\title{
ON THE FUNCTIONAL BLASCHKE-SANTALÓ INEQUALITY
}

\section{YOUJIANG LIN AND GANGSONG LENG}

Abstract. In this paper, using functional Steiner symmetrizations, we show that Meyer and Pajor's proof of the Blaschke-Santaló inequality can be extended to the functional setting.

Mathematics subject classification (2010): 46E30, 52A40.

Keywords and phrases: Steiner symmetrizations, Log-concave functions, Blaschke-Santaló inequality, Legendre transform.

\section{REFERENCES}

[1] S. Artstein, B. Klartag, V. D. Milman, On the Santaló point of a function and a functional Santaló inequality, Mathematika 54 (2004), 33-48.

[2] K. BALL, Isometric problems in $l_{p}$ and sections of convex sets, Doctoral thesis, University of Cambridge, 1986.

[3] K. BALL, Logarithmically concave functions and sections of convex sets in $\mathbb{R}^{n}$, Studia Math. 88 (1988), 69-84.

[4] W. BlaschKe, Über affine Geometrie 7: Neue Extremeigenschaften von Ellipse und Ellipsoid, Wilhelm Blaschke Gesammelte Werke 3, Thales Verlag, Essen (1985).

[5] H. J. Brascamp, E. H. Lieb, J. M. LutTinger, A General Rearrangement Inequality for Multiple Integrals, J. Funct. Anal. 17 (1974), 227-237.

[6] A. Burchard, Steiner symmetrization is continuous in $W^{1, p}$, Geom. Funct. Anal. 7 (1997), 823 860.

[7] A. BURChARD, A short course on rearrangement inequalities, available at http://www.math. utoronto.ca/almut/rearrange.pdf, 2009.

[8] M. FORTIER, Convergence results for rearrangements: Old and new, M.S. Thesis, University of Toronto, December 2010.

[9] M. Fradelizi, M. Meyer, Some functional forms of Blaschke-Santaló inequality, Math. Z. 256 (2007), 379-395.

[10] J. LEHEC, The symmetric property $\tau$ for the Gaussian measure, Ann. Fac. Sci. Toulouse Math. 17 (6) (2008), 357-370.

[11] J. LEHEC, Partitions and functional Santaló inequality, Arch. Math. 92 (2009), 89-94.

[12] J. LEHEC, A direct proof of the functional Santaló inequality, C. R. Math. Acad. Sci. Paris, 347 (2009), $55-58$.

[13] E. Lutwak, G. Zhang, Blaschke-Santaló inequalities, J. Differ. Geom. 47 (1) (1997), 1-16.

[14] E. Lutwak, D. Yang, G. Zhang, Moment-entropy inequalities, Ann. Probab. 32 (2004), 757-774.

[15] M. Meyer, A. Pajor, On the Blaschke Santaló inequality, Arch. Math. 55 (1990), 82-93.

[16] A. PRÉKOPA, Logarithmic concave measures with applications to stochastic programming, Acta Sci. Math. (Szeged) 32 (1971), 301-316.

[17] A. PrÉKopa, On logarithmic concave measures and functions, Acta Sci. Math. (Szeged) 34 (1973), 339-343.

[18] L. A. SANTALó, An affine invariant for convex bodies of $n$-dimensional space, Port. Math. 8 (1949), $155-161$.

[19] R. SchneIder, Convex Bodies: The Brunn-Minkowski Theory, Encyclopedia Math. Appl., vol. 44, Cambridge University Press, Cambridge, 1993. 